DOI : 10.5050/KSNVE.2010.20.1.083

$$
\begin{gathered}
\text { 이산계와 탄성 지지보의 동응답 및 진동 인텐시티 } \\
\text { 저감을 위한 목적함수 해석 }
\end{gathered}
$$

\title{
Analyses of the Cost function for the Reductions of the Dynamic Response and the Vibrational Intensity of a Discrete System and Its Elastic Supporting Beam
}

\author{
김 기 만†.최 성 대* \\ Gi Man Kim and Seong Dae Choi \\ (2009년 10월 22일 접수 ; 2009년 12월 15일 심사완료)
}

Key Words : Cost Function(목적함수), Dynamic Response(동응답), Vibrational Intensity(진동인텐시티), Feedforward Control(선먹임제어), Input Power(입력파워)

\begin{abstract}
In this paper, the feasibility of the cost function having two control factors were discussed in compared to two others which has one different control factor respectively. As of the control factors, the dynamic response of a discrete system and the vibrational intensity at the reference point which is the connecting point of a discrete system to a flexible beam were controlled actively by the control force obtained from the minimization of the cost function. The method of feedforward control was employed for the control strategy. The reduction levels of the dynamic response of a discrete system and the vibrational intensity at a reference point, and also the input power induced by the control force were evaluated numerically in cases of the three different cost functions. In comparison with the results obtained from the cost functions of one control factor, which is the dynamic response or the vibrational intensity, in most cases of the cost function of two control factors the better or similar results were obtained. As a conclusion, it is surely noted that both the dynamic response and the vibrational intensity of the vibrating system be controlled up to the expected level by using the single cost function having two control factors.
\end{abstract}

\section{1. 서 론}

산업 현장은 물론 거의 모든 부분에서 직접 또는 간접적으로 진동과 소음을 경험하고 있고 그에 대 한 부작용도 날이 갈수록 심해지고 있는 실정이다. 따라서 진동과 소음에 대한 원인 분석 및 제어를

† 교신저자; 정회원, 금오공과대학교 기계공학부

E-mail : giman@kumoh.ac.kr

Tel : (054)478-7294, Fax : (054)478-7319

* 금오공과대학교 기계공학부
위한 연구는 지속적으로 진행되고 많은 결과들이 현장에 적용되고 있다. 어느 특정 부위의 반복운동 에 의해 발생하는 진동 현상은 주변 구조물을 통해 진동에너지를 전달하게 되고 그 결과는 동응답과 고체전파소음으로 나타난다.

일반 구조물이나 기계의 경우 구성 요소로 보, 평판, 셸 모양의 단순 구조의 복합으로 형성되었다 고 가정하고 진동 구조물로 보, 평판, 셸에 대한 진 동 특성을 연구한 사례는 이미 상당 기간 지속되어 왔다. 특히 진동에너지나 파워 흐름을 분석하여 제 
어에 적용하는 경우가 늘고 있다. 보나 평판에서 전 달되는 에너지에 대한 크기와 방향을 표시하는 진 동인텐시티 측정법 ${ }^{(1)}$ 이 보고된 이후 1 차원 진동 모 델인 보에 대한 여러 경우에 대해 진동 인텐시티 해석 및 제어기술(2 7)이 수행 되었다. 연결 상태에 따라 전체 구조물의 모양이 2,3 차 구조물을 이루 는 복합 구조형태의 보 구조물인 프레임 ${ }^{(8-11)}$ 에 대 한 진동 파워 분석과 제어기법이 보고되었다. 다양 한 형태와 조건에 따른 평판에 대한 진동 파워 흐 름 ${ }^{(12 ~ 21)}$ 을 해석한 결과가 최근까지 나오고 있다.

이 연구에서 단순지지단을 지닌 탄성 보와 기준 점에 연결된 1 자유도 이산계의 진동 모델을 적용 하여 파워 흐름에 대한 기준점과 이산계의 고체전 파소음 및 동응답 저감을 동시에 달성하기 위해 세 종류의 목적함수를 비교 분석하여 그 적용 가능성 에 대해 검증하였다. 조화 가진력 $\left(F_{s}\right)$, 불균형 질량 점 $(m), 1$ 자유도 이산계(질량 $\left(M_{c}\right)$, 스프링 $(K)$, 댐퍼 $(C))$ 가 존재하는 지지 탄성 보의 횡진동 운동은 Bernoulli-Euler theory ${ }^{(22)}$ 를 근거로 최종 운동은 매 개변수분리법 ${ }^{(23)}$ 을 사용하여 해석하였다. 가진력을 단일 주파수의 단위 수직력으로 가정하여 선먹임 제어기법을 적용하였다. 선먹임제어기법은 되먹임제 어기법(feedback control method)과 같이 다양한 가 진주파수대에서 적용은 어렵지만 일정한 가진주파 수에 사용이 편리한 것으로 이미 많은 연구와 검증 결과를 도출한 기법이다. Tanaka ${ }^{(15)}$ 는 사각평판의 진동제어를 위해 가진력과 제어력의 위상차를 이용 하여 전달되는 파워의 최소화를 구현하였고 $\mathrm{Nam}^{(16)}$

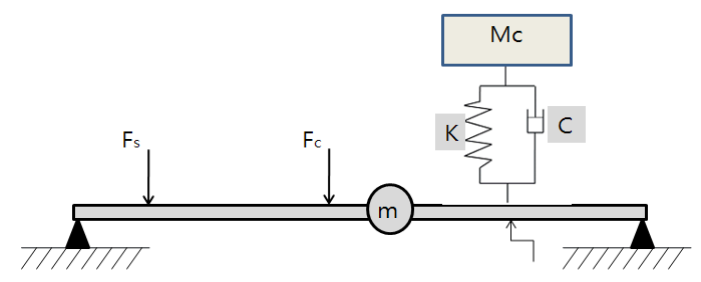

$\mathrm{Rp}_{\mathrm{p}}$

Fig. 1 Theoretical model of a compound vibrating system with a single degree of freedom system and a elastic supporting beam: $F_{s}$ (the primary force), $F_{c}$ (the control force), $m$ (an irregular point mass), $R_{p}$ (a reference point), a single degree of freedom system( control mass $M_{c}$, spring stiffness $K$, damping constant $C$ )
은 단순 사각평판의 국소점에 진동인텐시티 최소화 를 선먹임제어기법을 적용하여 연구하였다. 또한 $\mathrm{Bardou}^{(17)}$ 유한 무한 평판에 파워 흐름을 저감을 실 현하였다. 최적 제어력을 구하기 위해 급강하법을 통해 목적함수 최소화를 실행하였다. Fig. 1은 단순 지지대의 탄성 보와 여러 주변 조건을 나타내는 이 론적 모델을 보여 주고 있다.

\section{2. 기본방정식 전개}

\section{1 운동방정식 유도}

가진력, 제어력, 1 자유도 이산계, 질량점을 지닌 탄성 지지보의 운동방정식은 아래와 같이 나타난다.

$$
\begin{aligned}
& E I \frac{\partial^{4} W(x, t)}{\partial x^{4}}+\left(\rho A+m \delta\left(x-x_{2}\right)\right) \frac{\partial^{2} W(x, t)}{\partial t^{2}} \\
& =\left[\begin{array}{c}
C\left\{\frac{d Y(t)}{d t}-\frac{\partial W(x, t)}{\partial t}\right\} \\
+K\{Y(t)-W(x, t)\}
\end{array}\right] \delta\left(x-x_{1}\right) \\
& +F_{s} e^{i \omega t} \delta\left(x-x_{s}\right)+F_{c} e^{i \omega t} \delta\left(x-x_{c}\right)
\end{aligned}
$$

여기서 $W(x, t)$ 는 보의 변위, $Y(t)$ 는 이산계 변위, $\omega$ 는 가진주파수, $x_{s}$ 는 가진력의 위치, $x_{c}$ 는 제어기 위 치, $x_{2}$ 는 질량점의 위치, $x_{1}$ 은 이산계의 위치 및 진 동인텐시티 제어를 위한 기준점, $\delta($ )는 Dirac delta 함수를 각각 나타낸다.

1 자유도 이산계의 운동은 지지보의 반력을 고려 하여 다음과 같이 정의된다.

$$
\begin{aligned}
& M_{c} \frac{d^{2} Y(t)}{d t^{2}}+C \frac{d Y(t)}{d t}+K Y(t) \\
& =K W(x, t) \delta\left(x-x_{1}\right) \\
& \quad+C \frac{\partial W(x, t)}{\partial t} \delta\left(x-x_{1}\right)
\end{aligned}
$$

조화가진력에 의해 지지보와 이산계의 운동을 조 화운동으로 정의하면 다음과 같다.

$$
W(x, t)=w(x) e^{i \omega t} \text { and } Y(t)=y e^{i \omega t}
$$

여기서 $w(x)$ 와 $y$ 는 각각 지지보와 이산계 질량의 처짐을 나타낸다. 따라서 시간 항을 소거한 식 (1)의 완전해는 제차해 $\left(w_{h}\right)$ 와 특해 $\left(w_{p}\right)$ 의 합으로 다음과 
같다.

$$
w(x)=w_{h}+w_{p}
$$

여기서 제차해는

$$
\begin{aligned}
w_{h}(x)= & H_{1} \cos (\beta x)+H_{2} \sin (\beta x)+ \\
& H_{3} \cosh (\beta x)+H_{4} \sinh (\beta x)
\end{aligned}
$$

여기서 상수 $\left(H_{1 \sim 4}\right)$ 는 단순지지 경계조건에 의해 최 종 식(4)에서 얻어지고 특해는 매계변수분리법을 이용하여 다음과 같이 가정한다.

$$
\begin{aligned}
w_{p}(x)= & V_{1}(x) \cos (\beta x)+V_{2}(x) \sin (\beta x)+ \\
& V_{3}(x) \cosh (\beta x)+V_{4}(x) \sinh (\beta x)
\end{aligned}
$$

여기서 파동수는 $\beta=\sqrt[4]{\frac{\rho A \omega^{2}}{E I}}, V_{1 \sim 4}$ 는 특해 계 수로 다음과 같이 정의한다.

$$
\begin{aligned}
& V_{1}(x)=\frac{1}{2 \beta^{3}} \int^{x} \sin (\beta x) f(\eta) d \eta \\
& V_{2}(x)=-\frac{1}{2 \beta^{3}} \int^{x} \cos (\beta x) f(\eta) d \eta \\
& V_{3}(x)=-\frac{1}{2 \beta^{3}} \int^{x} \sinh (\beta x) f(\eta) d \eta \\
& V_{4}(x)=\frac{1}{2 \beta^{3}} \int^{x} \cosh (\beta x) f(\eta) d \eta
\end{aligned}
$$

여기서 $f(\eta)$ 힘함수(forcing function)를 나타내며,

$$
\begin{aligned}
f(\eta)= & \frac{F_{s}}{E I} \delta\left(\eta-x_{s}\right) \\
& +\frac{F_{c}}{E I} \delta\left(\eta-x_{c}\right)+\frac{m \omega^{2}}{E I} \delta\left(\eta-x_{2}\right) \\
& +\frac{M_{c} \omega^{2}(K+i \omega C) w\left(x_{1}\right) \delta\left(\eta-x_{1}\right)}{E I\left\{\left(K-M_{c} \omega^{2}\right)+i \omega C\right\}}
\end{aligned}
$$

식 (8)을 식 (7)에 대입하여 각 계수를 정리한 후 식 (4)에 단순지지 경계조건을 적용하면 상수 $H_{1}$ 과 $\mathrm{H}_{3}$ 는 영이 되고 최종 해는 계단함수로 정의된 힘의 영향계수를 포함하여 다음과 같이 얻어진다.

$$
\begin{aligned}
w(x)= & H_{2}\left\{\sin (\beta x)+h_{21} M_{1}(x)+h_{22} M_{2}(x)\right\} \\
& +H_{4}\left\{\sinh (\beta x)+h_{41} M_{1}(x)+h_{42} M_{2}(x)\right\} \\
& +P_{s}(x)+P_{c}(x)+h_{51} M_{1}(x)+h_{52} M_{2}(x)
\end{aligned}
$$

여기서

$$
\begin{aligned}
& H_{2}=\frac{B_{3} B_{5}-B_{2} B_{6}}{B_{1} B_{5}-B_{2} B_{4}}, \\
& H_{4}=-\frac{B_{3} B_{4}-B_{1} B_{6}}{B_{1} B_{5}-B_{2} B_{4}}
\end{aligned}
$$

그리고 각 힘의 영향계수는 아래와 같다.

$$
\begin{aligned}
& M_{1}(x)=\left[\frac{-M_{c} \omega^{2}(K+i \omega C)}{2 \beta^{3} E I\left\{\left(K-M_{c} \omega^{2}\right)+i \omega C\right\}}\right], \\
& *\left\{\sin \left(\beta\left(x-x_{1}\right)\right)-\sinh \left(\beta\left(x-x_{1}\right)\right)\right\} U\left(x-x_{1}\right)
\end{aligned}
$$

$$
\begin{aligned}
M_{2}(x)= & {\left[\frac{-m \omega^{2}}{2 \beta^{3} E I}\right]\left\{\sin \left(\beta\left(x-x_{2}\right)\right)\right.} \\
& \left.-\sinh \left(\beta\left(x-x_{2}\right)\right)\right\} U\left(x-x_{2}\right)
\end{aligned}
$$

$$
\begin{aligned}
P_{s}(x)= & {\left[\frac{-F_{s}}{2 \beta^{3} E I}\right]\left\{\sin \left(\beta\left(x-x_{s}\right)\right),\right.} \\
& \left.-\sinh \left(\beta\left(x-x_{s}\right)\right)\right\} U\left(x-x_{s}\right) \\
P_{c}(x)= & {\left[\frac{-F_{c}}{2 \beta^{3} E I}\right]\left\{\sin \left(\beta\left(x-x_{c}\right)\right)\right.} \\
& \left.-\sinh \left(\beta\left(x-x_{c}\right)\right)\right\} U\left(x-x_{c}\right),
\end{aligned}
$$

$$
\begin{aligned}
& h_{21}=\sin \left(\beta x_{1}\right)+M_{2}\left(x_{1}\right) \sin \left(\beta x_{2}\right), \\
& h_{22}=\sin \left(\beta x_{2}\right)+M_{1}\left(x_{2}\right) \sin \left(\beta x_{1}\right), \\
& h_{41}=\sinh \left(\beta x_{1}\right)+M_{2}\left(x_{1}\right) \sinh \left(\beta x_{2}\right), \\
& h_{42}=\sinh \left(\beta x_{2}\right)+M_{1}\left(x_{2}\right) \sinh \left(\beta x_{1}\right), \\
& h_{51}=P_{c}\left(x_{1}\right)+P_{s}\left(x_{1}\right)+M_{2}\left(x_{1}\right)\left(P_{c}\left(x_{2}\right)+P_{s}\left(x_{2}\right)\right), \\
& h_{52}=P_{s}\left(x_{2}\right)+P_{c}\left(x_{2}\right)+M_{1}\left(x_{2}\right)\left(P_{c}\left(x_{1}\right)+P_{s}\left(x_{1}\right)\right) \\
& B_{1}=\sin (\beta L)+h_{21} M_{1}(L)+h_{22} M_{2}(L) \\
& B_{2}=\sinh (\beta L)+h_{41} M_{1}(L)+h_{42} M_{2}(L), \\
& B_{3}=P_{s}(L)+P_{c}(L)+h_{51} M_{1}(L)+h_{52} M_{2}(L) \\
& B_{4}=-\beta^{2} \sin (\beta L)+h_{21} M_{1}^{\prime \prime}(L)+h_{22} M_{2}^{\prime \prime}(L) \\
& B_{5}=\beta^{2} \sinh (\beta L)+h_{41} M_{1}^{\prime \prime}(L)+h_{42} M_{2}^{\prime \prime}(L), \\
& B_{6}=P_{s}^{\prime \prime}(L)+P_{c}^{\prime \prime}(L)+h_{51} M_{1}^{\prime \prime}(L)+h_{52} M_{2}^{\prime \prime}(L) .
\end{aligned}
$$

여기서 "'은 $x$ 에 대한 2계 미분을 함수 $U()$ 는 계 단함수를 의미한다.

\section{2 목적함수 정의}

이 연구에서는 이산계와 탄성 지지보로 구성된 
복합진동계의 진동 저감을 위해 복수 제어인자를 지닌 목적함수에서 얻어진 최적 제어 조건들을 단 수 제어 인자를 지닌 두 목적함수들의 결과와 비교 하여 목적함수의 신뢰 정도를 검토하였다. 제어인자 로서는 이산계 질량의 동응답(dynamic response of a $\operatorname{mass}(D M) ; y)$ 및 기준점(이산계와 지지보 연결 점)에 전달되는 고체전파소음을 표현하는 진동인텐 시티(vibrational intensity $(V I) ; I(x))$ 를 사용하여 복 수 또는 각각의 단수 인자를 지닌 목적함수를 다음 과 같이 정의한다.

$$
\begin{aligned}
& \Pi_{D M+V I}=(\operatorname{Real}[y])^{2}+\left(\operatorname{Real}\left[I\left(x_{1}\right)\right]\right)^{2} \\
& \Pi_{D M}=(\operatorname{Real}[y])^{2} \\
& \Pi_{V I}=\left(\operatorname{Real}\left[I\left(x_{1}\right)\right]\right)^{2}
\end{aligned}
$$

여기서 이산계 질량 동응답과 기준점에 전달되는 진동 인텐시티는 아래의 식으로 나타낸다.

$$
\begin{aligned}
& y=\frac{(i \omega C+K) w\left(x_{1}\right)}{\left(K-M_{c} \omega^{2}\right)+i \omega C} \\
& I(x)=-i \omega E I\left[\frac{\partial^{3} w(x)}{\partial x^{3}} w^{*}(x)-\frac{\partial^{2} w(x)}{\partial x^{2}} \frac{\partial w^{*}(x)}{\partial x}\right]
\end{aligned}
$$

여기서 '*’은 공액 복소수를 나타낸다. 식 (15)는 실 수와 허수로 구분되며 실수는 실제 전달되는 파워 (active intensity)의 크기를 허수는 반향파워(reactive intensity) 크기를 각 각 나타낸다. 따라서 제어 인 자를 최소화하기 위한 제어력은 가진력과 위상차를 고려한 복소수 형태로 주어지고 급강하법을 적용하 여 목적함수 값을 최소화하는 과정은 다음과 같이 정의한다.

$$
\nabla_{f} \Pi \Rightarrow \text { Minimization of } \Pi\left(f_{c} \rightarrow f_{\text {optimal }}\right)
$$

여기서 $f_{c}\left(=f_{r}+i f_{i}\right)=\frac{F_{c} L^{2}}{E I}$. 최적 제어력은 mathmatica의 최소값 판별(FindMinimum) 프로그램 을 이용하여 구하였다.

\section{3. 수치 계산 결과 및 분석}

수치 계산의 모든 결과를 무차원 값으로 표현하
였다. 모든 위치나 변위는 보의 길이 $(L)$ 의 대비로 표현하였고 힘의 경우는 단위 면적 당 보의 굽힘 강도 $\left(E I / L^{2}\right)$ 를, 질량은 보의 질량 $(\rho A L)$ 대비를 각 각 사용하여 결과를 구하였다. 주파수는 파동수에 보의 길이를 곱한 무차원주파수 $(\alpha)$ 를 적용하였다. 따라서 결과에 나타나는 제어 인자들의 값들도 기 본 물성치를 근거로 무차원화 하였으며 사용한 기 본 물성치에 대한 무차원 형태와 값이 Table 1 에 기 술되어있다.

Table 1 Values of physical properties

\begin{tabular}{c|c|c}
\hline \hline & Dimensionless expression & Value \\
\hline $\begin{array}{c}\text { A discrete system } \\
\text { mass }\end{array}$ & $m_{1}=\frac{M_{c}}{\rho A L}$ & 0.5 \\
\hline $\begin{array}{c}\text { A discrete system } \\
\text { spring stiffness }\end{array}$ & $k=\frac{K L^{3}}{E I}$ & 1000 \\
\hline $\begin{array}{c}\text { A discrete system } \\
\text { damping ratio }\end{array}$ & $\xi$ & 0.5 \\
\hline Source location & $X_{s}=x_{s} / L$ & 0.3 \\
\hline $\begin{array}{c}\text { Primary force } \\
\text { An irregular mass }\end{array}$ & $f_{s}=\frac{F_{s} L^{2}}{E I}$ & 1 \\
\hline $\begin{array}{c}\text { An irregular mass } \\
\text { location }\end{array}$ & $m_{2}=\frac{m}{\rho A L}$ & 0.3 \\
\hline $\begin{array}{c}\text { Structural damping } \\
\text { A discrete system } \\
\text { mass location }\end{array}$ & $X_{2}=x_{2} / L$ & 0.5 \\
\hline \begin{tabular}{c} 
Controller location \\
\hline
\end{tabular} & $X_{1}=x_{1} / L$ & 0.001 \\
\hline
\end{tabular}

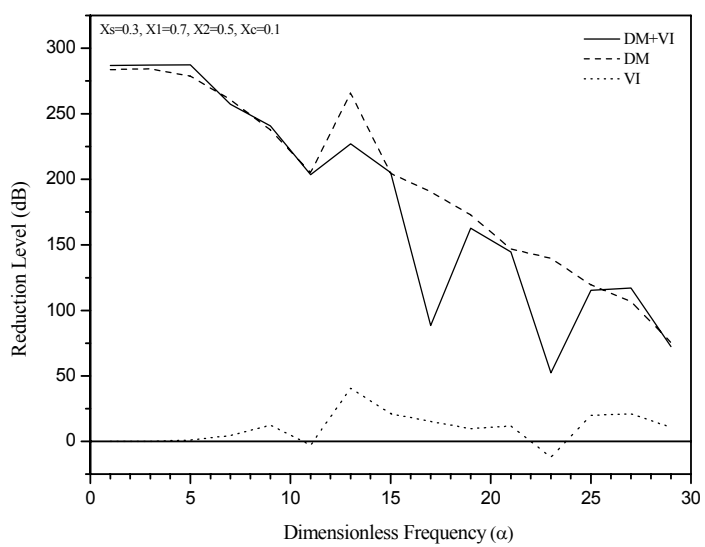

Fig. $2 D M$ reduction vs. $\alpha$; $X_{c}=0.1, X_{s}=0.3, X_{2}$ $=0.5, X_{1}=0.7$, Solid (Eq.(11)), Dash(Eq.(12)), $\operatorname{Dot}($ Eq.(13)) 


\section{1 동응답 저감}

탄성 지지보에 연결된 이산계 질량의 동응답 $(D M)$ 제어를 위해 세 종류의 목적함수 최소화를 통해 얻 어진 결과를 제어 전 값과 비교하여 저감 정도를 분석하였다. 대비 식은 다음과 같다.

$$
D M \text { reduction }=20 \log _{10}\left|\frac{\operatorname{Real}[y]_{\text {before }}}{\operatorname{Real}[y]_{\text {after }}}\right|
$$

Fig. 2는 목적함수 최소화에서 얻어진 최적 제어력 을 사용하여 무차원 주파수 $(\alpha)$ 에 따라 나타나는 이 산계 동응답 저감 정도를 보여 주고 있다. 제어인자 로 동응답을 포함하는 두 목적함수(Eq. (11) - DM+ $V I$, Eq.(12) - $D M$ )의 경우는 결과가 비슷한 형태로 크 게는 $300 \mathrm{~dB}$ 에서 작게는 $100 \mathrm{~dB}$ 정도의 저감을 보여 주고 전반적으로 주파수대가 커질 수 록 저감 정도 가 작아지는 경향을 보여 주고 있다. 하지만 진동인 텐시티만 고려한 목적함수(Eq. (13) - VI)는 주파수가 커질수록 $20 ~ 30 \mathrm{~dB}$ 의 저감 효과를 보여주고 있으나 전반적으로 미미한 것으로 나타났다.

Fig. 3에서는 고정주파수 $(\alpha=9)$ 에서 제어기 위 치에 따른 세 경우의 이산계 위치 $\left(X_{1}=0.1,0.4\right.$, 0.7)에 대하여 동응답 저감을 조사하였다. 세 경우 모두 큰 감소를 보여주고 동응답 제어인자를 지닌 두 목적함수의 결과가 거의 같은 형태로 나타나고 있다. 진동인텐시티만의 목적함수 사용은 동응답 제 어에는 그 효과가 매우 미미한 것으로 나타났지만 두 제어인자를 지닌 목적함수의 사용은 충분한 효 과를 얻을 수 있는 것으로 판단된다.

\section{2 진동 인텐시티 저감}

가진력에 의해 전달되는 고체전파소음의 저감 정 도를 이산계 연결부를 기준점으로 하여 제어 전 (before)과 제어 후(after) 진동 인텐시티 $(V I)$ 를 비교 한 아래의 식으로 표현하였다.

$$
V I \text { reduction }=10 \log _{10}\left|\frac{V I_{\text {before }}}{V I_{\text {after }}}\right|
$$

Fig. 4은 무차원 주파수대에 따라 기준점 $\left(R_{p}=\right.$ $\left.X_{1}\right)$ 에 전달되는 진동인텐시티 감소 정도를 보여 주 고 있다. 앞 절의 동응답 결과와 비슷한 현상을 보 여주고 있으나 전반적으로 저감 크기가 $100 \mathrm{~dB}$ 정 도 줄어들고 목적함수 경우는 두 목적함수 (Eq.
(12) - $D M$, Eq. (13) - VI)가 반대의 효과를 나타내 고 있다.

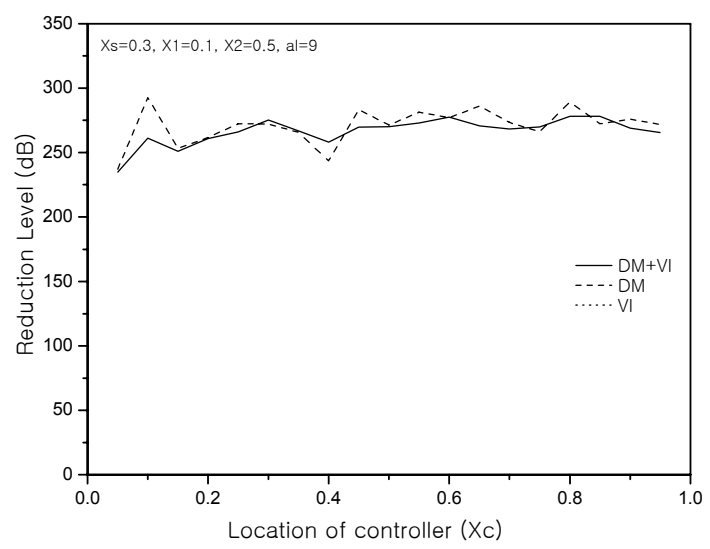

(a) $X_{1}=0.1$

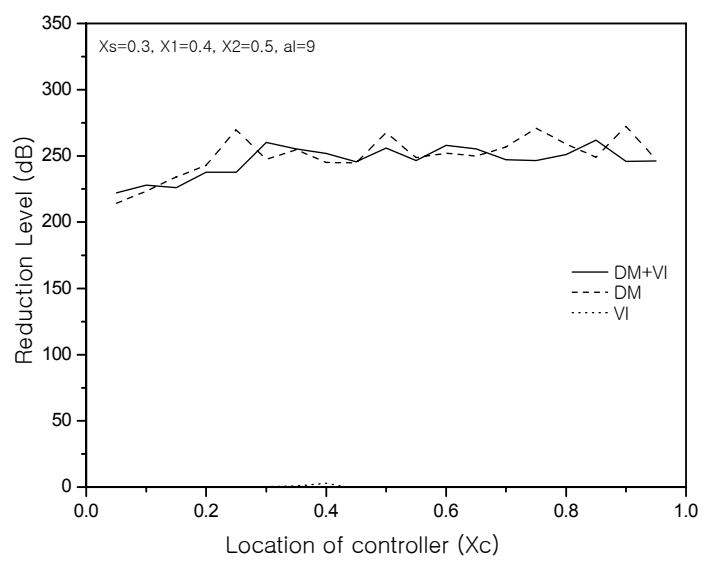

(b) $X_{1}=0.4$

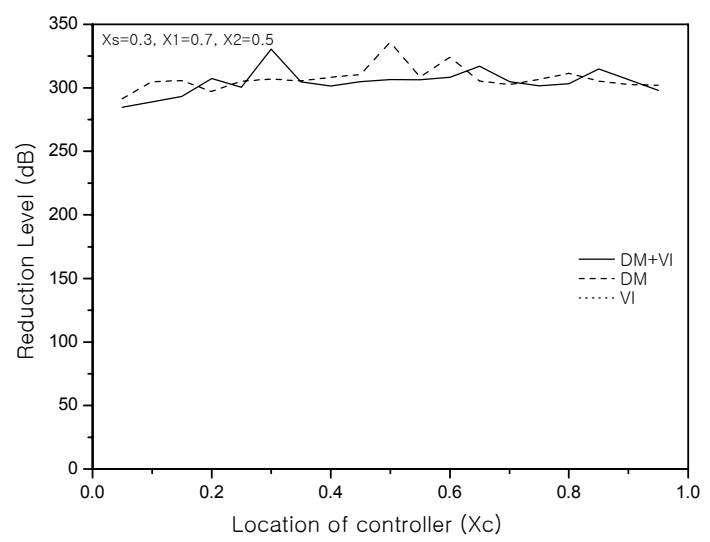

(c) $X_{1}=0.7$

Fig. $3 D M$ reduction vs. $X_{c} ; \alpha=9, X_{s}=0.3, X_{2}=0.5$, Solid (Eq. (11)), Dash(Eq. (12)), Dot(Eq. (13)) 


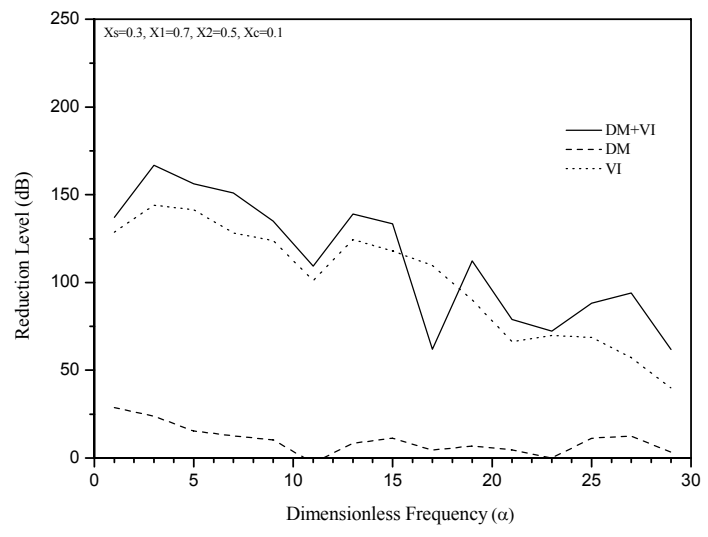

Fig. 4 VI reduction vs. $\alpha$; $X_{c}=0.1, X_{s}=0.3, X_{2}=$ 0.5, $X_{1}=0.7$, Solid(Eq. (11)), Dash(Eq. (12)), $\operatorname{Dot}(E q .(13))$

Fig. 5에서는 고정주파수 $(\alpha=9)$ 에서 제어기 위치 에 따른 세 경우의 이산계 위치 $\left(X_{1}=0.1,0.4,0.7\right)$ 에 대하여 진동인텐시티 저감을 조사하였다. 세 경우 모 두 큰 감소를 보여주고 진동인텐시티 제어인자를 지 닌 두 목적함수(Eq. (11) - DM+VI, Eq. (13) - VI)의 결 과가 거의 같은 형태로 나타나고 있다. 동응답 제어 인자 목적함수(Eq. (12)) 사용은 진동인텐시티 증가를 초래하는 경우도 발생하는 것으로 나타났다.

\section{3 고찰 및 검토}

하나의 수직 제어력을 사용하여 기준점 진동인텐 시티 및 이산계의 동응답을 동시에 제어하고자 하 는 목적함수의 효과를 검토하였다. 제어인자로 동응 답과 진동인텐시티 중 하나만을 고려한 목적함수의 결과와 비교하여도 동시에 두 제어인자를 지닌 목 적함수 사용 시 기준점에 대한 동응답 및 진동인텐 시티 저감에 만족스러운 결과를 도출하는 것으로 나타났다. 하지만 특정 영역 이외 전체 시스템 입장 에서 제어에 따른 진동 특성의 변화 및 제어기 성 능에 대한 검토가 최적 제어 조건에 포함 되어야 된다고 본다. Fig. 6은 제어기 입력파워 변화를 무차 원 주파수에 따라 조사한 결과다. 제어기 입력파워 는 아래의 식으로 나타난다.

$$
I P=10 \log _{10}\left|\operatorname{Real}\left[\frac{1}{2} f_{c} \frac{\partial W^{*}\left(x_{1}, t\right)}{\partial t}\right]\right|
$$

값이 작을 수 록 입력파워가 작게 사용되는 것으

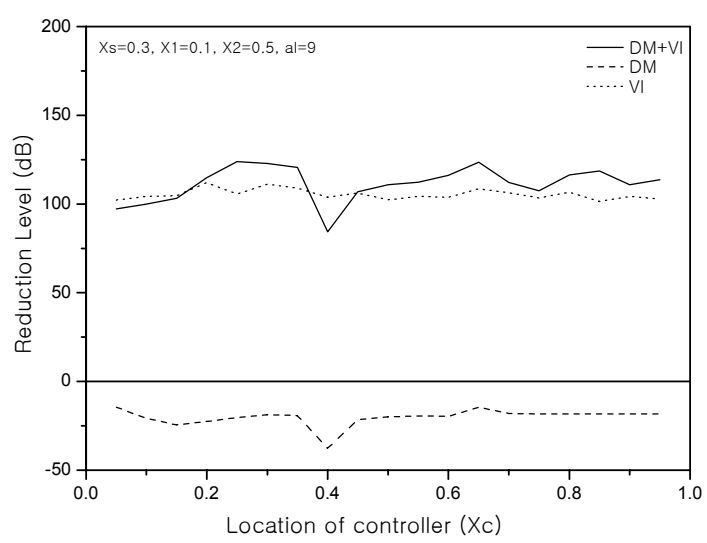

(a) $X_{1}=0.1$

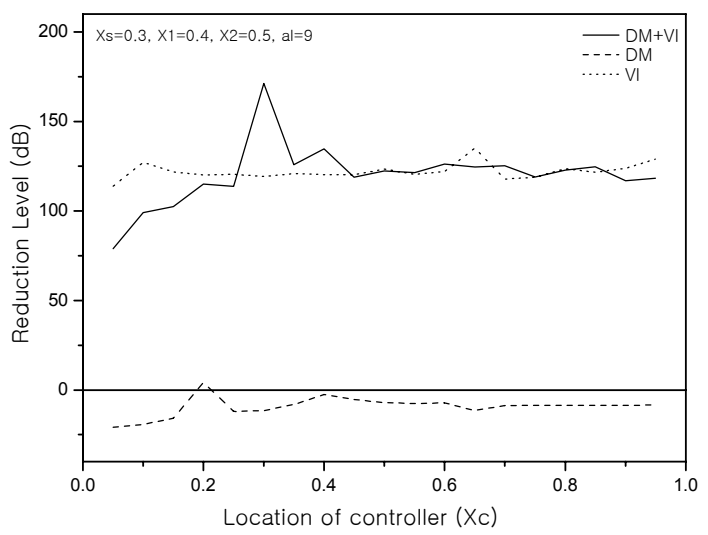

(b) $X_{1}=0.4$

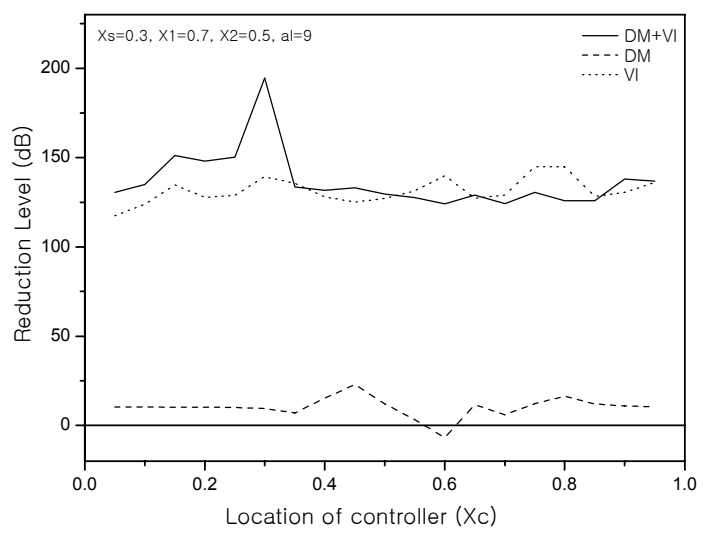

(c) $X_{1}=0.7$

Fig. $5 V I$ reduction vs. $X_{c} ; \alpha=9, X_{c}=0.3, X_{2}=$ 0.5, Solid (Eq. (11)), Dash(Eq. (12)), Dot(Eq. (13))

로 전체 주파수대에서 두 제어인자 목적함수의 결 과가 다른 두 경우에 비해 작게 나타나고 있다. 


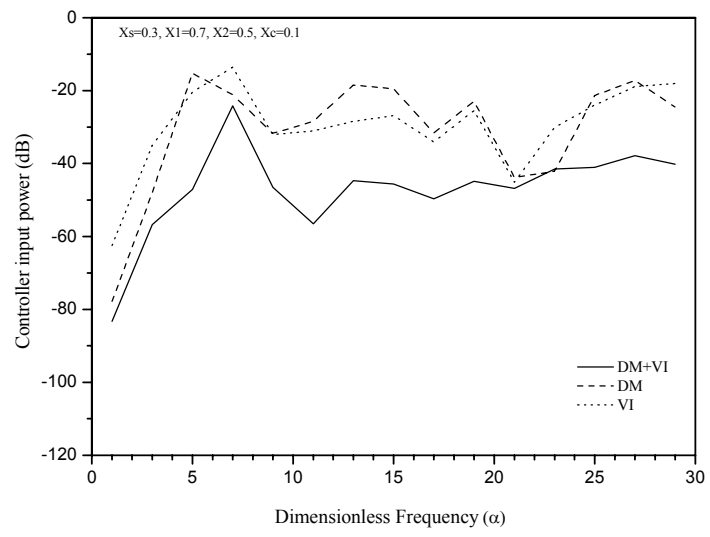

Fig. 6 Input power of controller vs. $\alpha ; X_{c}=0.1, X_{s}=$ 0.3, $X_{2}=0.5, X_{1}=0.7$, Solid(Eq. (11)), Dash(Eq. (12)), $\operatorname{Dot}($ Eq. (13))

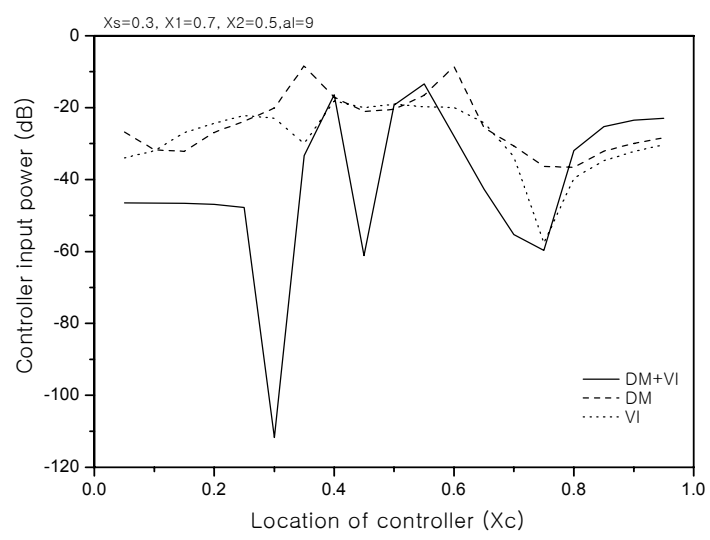

Fig. 7 Input power of Controller vs. $X_{c} ; \alpha=9, X_{s}=$ 0.3, $X_{2}=0.5, X_{1}=0.7$, Solid(Eq. (11)), Dash(Eq. (12)), $\operatorname{Dot}($ Eq. (13))

Fig. 7은 고정주파수 $(\alpha=9)$ 에서 제어기 위치 변화에 따른 입력파워 크기를 보여주고 있다. 전반적으로 두 제어인자 목적함수의 결과가 다른 두 경우에 비 해 좋은 것으로 나타나고 있다.

지지 및 전달 매체 역할을 하는 탄성보의 전체 동응답과 진동인텐시티 분포에 대한 조사를 수행하 였다. Fig. 8은 기준점 이산계 동응답 저감을 위한 동응답 제어인자를 지닌 두 경우의 목적함수에서 얻어진 제어 후와 제어 전 보 전체 처짐을 비교하 여 보여 주고 있다. 두 목적함수 사용에 따른 결과 가 비슷한 형태로 나타나고 제어 전에 비해 처짐이 작아 졌다.

Fig. 9는 진동인텐시티 제어인자를 지닌 두 목적

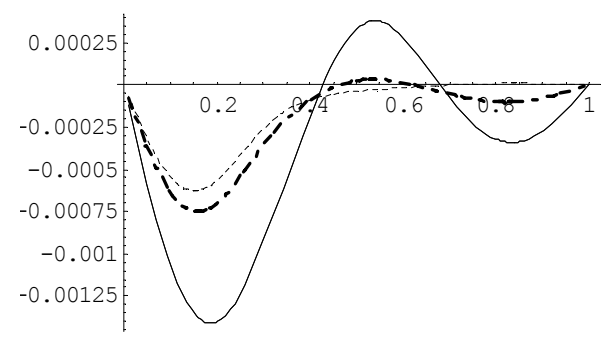

Fig. 8 Beam deflection along its length; $\alpha=9, X_{c}=$ $0.1, X_{s}=0.3, X_{2}=0.5, X_{1}=0.7$, Thin(Eq. (11)), Thick(Eq. (12)), Soild(before-control)

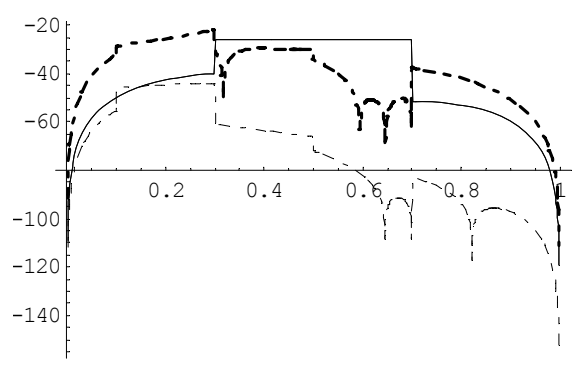

Fig. 9 Vibrational intensity along beam length; $\alpha=$ $9, \quad X_{c}=0.1, \quad X_{S}=0.3, \quad X_{2}=0.5, \quad X_{1}=0.7$ Thin(Eq. (11)), Thick(Eq. (13)), Soild(beforecontrol)

함수에 따른 진동인텐시티 분포를 보여 주고 있다. 두 제어인자 목적함수(Eq. (11))의 분포 정도가 제어 전에 비해 전반적으로 작게 형성이 되었고 한 제어 인자 목적함수(Eq. (13))의 경우와 비교하여도 더 좋 은 결과를 보여 주고 있다.

\section{4. 결 론}

이 연구에서는 두 제어인자(이산계 동응답 및 기 준점 진동인텐시티)를 하나의 제어력을 사용하여 저 감하기 위해 정의된 목적함수의 신뢰 정도를 비교 분석하였다. 비교 대상으로 두 제어인자(이산계 동 응답 및 기준점 진동인텐시티) 중 한 제어인자를 지닌 목적함수를 사용하였다. 제어기법으로는 선먹 임기법을 적용하였다.

결론적으로 두 제어인자를 같이 지닌 목적함수의 모든 결과들이 한 제어인자 목적함수들 결과와 비 교해도 비슷한 수준에 이르는 것으로 나왔다. 따라 서 두 개의 다른 인자를 동시에 제어 할 경우 하나 
의 목적함수에 포함하여 최적 제어 조건을 구현 할 수 있음을 검증하였다. 하지만 이 연구에 제시한 이 론적 저감 수치는 실제 적용 시 예상되는 값과 차이 가 날 수 도 있으며 단지 전반적인 제어의 경향을 표시하는 것으로 판단된다. 또한 제어 인자 갯수가 늘어나거나 시스템 구조 변화(이산계 및 질량점 증 가, 경계조건 변화 등)에 따라 전반적으로 저감 정도 가 작아지거나 효과가 미미할 수 도 있다고 본다. 따 라서 차 후 여러 조건을 포함한 구체적인 구조물에 대한 제어를 위해 제어인자, 제어력 형태, 목적함수 구성에 연구가 더 필요 할 것으로 사료된다.

\section{후 기}

이 연구는 금오공과대학교 학술연구비 지원에 의 하여 수행되었습니다.

\section{참 고 문 헌}

(1) Noiseux, D. J., 1970, "Measurement of Power Flow in Uniform Beams and Plates," J. Acoust. Soc. Am., Vol. 47, No. 1, pp. 238 247.

(2) Pan, J. and Hansen, C. H., 1991, "Active Control of Total Vibratory Power Flow in a Beam. I : Physical System Analysis," J. Acoust. Soc. Am., Vol. 89, No. 1, pp. 200 209.

(3) Enelund, M., 1993, "Mechanical Power Flow and Wave Propagation in Infinite Beams on Elastic Foundations," Structural Intensity and Vibrational Energy Flow 4th International Congress on Intensity Techniques, pp. 231 238.

(4) Schwenk, A. E., Sommerfeldt, S. D. and Hayek, S. I., 1994, "Adaptive Control of Structural Intensity Associated with Bending Waves in a Beam," J. Acoust. Soc. Am., Vol. 96, No. 5, pp 2826 2835.

(5) Shankar, K. and Keane, A. J., 1995, "Energy Flow Predictions in a Structure of Rigidly Joined Beams Using Receptance Theory," Journal of Sound and Vibration, Vol. 185, No. 5, pp. 867 890.

(6) Le Bot, A., Ichchou, M. N. and Jezequel, L., 1997, "Energy Flow Analysis for Curved Beams," J.
Acoust. Soc. Am., Vol. 102, No. 2, pp. 943 954.

(7) Kim, G. M., 2002, "Active Control of Vibrational Intensity in a Compound Vibratory Sysyem," J. of the Korean Society of Precision Engineering, Vol. 19, No. 6, pp. 109 118.

(8) Horner, J. L., 1993, "Prediction of Vibrational Power Transmission in Frameworks," Structural Intensity and Vibrational Energy flow 4th International Congress on Intensity Techniques, pp. 239 246.

(9) Beale, L. S. and Accorsi, M. L., 1995, "Power Flow in Two- and Three-dimensional Frame Structures," Journal of Sound and Vibration, Vol. 185, No. 4, pp. 685 702.

(10) Farag, N. H. and Pan, J., 1996, "Dynamic Response and Power Flow in Two-dimensional Coupled Beam Structures under In-plane Loading," J. Acoust. Soc. Am., Vol. 99, No. 5, pp. 2930 2937.

(11) Farag, N. H. and Pan, J., 1997, "Dynamic Response and Power Flow in Three-dimensional Coupled Beam Structures. I. Analytical Modeling," J. Acoust. Soc. Am., Vol. 102, No. 1, pp. 315 325.

(12) Hambric, S. A., 1990, "Power Flow and Mechanical Intensity Calculation in Structural Finite Element Analysis," Journal of Vibration and Acoustics, Vol. 112, pp. 542 549.

(13) Bouthier, O., Bernhard, R. and Wohlever, C., 1990, "Energy and Structural Intensity Formulations of Beam and Plate vibrations," Structural Intensity and Vibrational Energy Flow 3rd International Congress on Intensity Techniques, pp. 37 44.

(14) Pan, J., 1992, "Total Power Flow from a Vibrating Rigid Body to a Thin Panel Through Multiple Elastic Mounts,” J. Acoust. Soc. Am 92, No. 2, pp. 895 907.

(15) Tanaka, N., Snyder, S. D., Kikushima, Y., and Kuroda, M., 1994, "Vortex Structural Power Flow in a Thin Plate and the Influence on the Acoustic Field," J. Acoust. Soc. Am. 96, No. 3, pp. 1563 1574.

(16) Nam, M., Hayek, S. I. and Sommerfeldt, S. D., 1995, "Active Control of Structural Intensity in 
Connected Structures," Proceedings of the Conference Active 95, pp. 209 220.

(17) Bardou, O., Gardonio, P., Elliott, S. J. and Pinnington, R. J., 1997, “Active Power Minimization and Power Absorption in a Plate with Force and Moment Excitation,” J. of Sound and Vibration, Vol. 208, No. 1, pp. 111 151.

(18) Park, D. H., Hong, S. Y. and Kil, H. G., 1998, "Vibration Power Flow Analysis of Coupled Co-planar Rectangular Plates," Transactions of the Korean Society for Noise and Vibration Engineering, Vol. 8, No. 6, pp. 1053 1061.

(19) Kim, G. M., 2001, "Active Control Vibrational Intensity at a Reference Point in an Infinite Elastic Plate," Transactions of the Korean Society for Noise and Vibration Engineering, Vol. 11, No. 4, pp. 22 30.
(20) Choi, J. S., Kil, H. G. and Hong, S. Y., 2001, "Power Flow Analysis of Vibration of Coupled Plates Excited by a Point Force In an Arbitrary Direction," Transactions of the Korean Society for Noise and Vibration Engineering, Vol. 18, No. 3, pp. 181 192.

(21) Lee, J. Y., Kil, H. G., Song, J. H. and Hong, S. Y., 2009, "Power Flow Analysis of Vibration of a Plate Covered with a Damping Sheet," Transactions of the Korean Society for Noise and Vibration Engineering, Vol. 19, No. 5, pp. 530 536.

(22) Junger, M. C. and Feit, D., 1985, "Sound, Structures and Their Interaction," The MIT Press, pp. 197 201.

(23) Hildebrand, F. B., 1976, "Advanced Calculus for Applications 2ed," Prentice-Hall, pp. 24 28. 